\title{
CUIDADOS PALIATIVOS: PERCEPÇÃO DE MÉDICOS QUE ATUAM NA ATENÇÃO PRIMÁRIA À SAÚDE
}

\author{
PALLIATIVE CARE: PERCEPTION OF DOCTORS WHO WORK IN \\ PRIMARY HEALTH CARE
}

\author{
Ana Luiza Alves Gurgel França ${ }^{1}$ \\ Luciana Modesto de Brito ${ }^{2}$ \\ Cícera Amanda Mota Seabra ${ }^{3}$ \\ Ankilma do Nascimento Andrade Feitosa ${ }^{4}$
}

\begin{abstract}
RESUMO: Introdução: O atual cenário epidemiológico implicou na mudança da assistência à saúde de forma que contemple todo o processo saúde-doença dos indivíduos. Neste sentido, os cuidados paliativos surgem como uma estratégia de continuar prestando cuidados às pessoas acometidas por doenças sem cura, de forma a garantir o conforto e qualidade de vida. A família também é incluída nesse cuidado, pois é quem vivencia mais de perto as modificações na vida do indivíduo. $A$
\end{abstract}

\footnotetext{
${ }^{1}$ Médica. Residente em Saúde da Família e Comunidade da Faculdade Santa Maria, Cajazeiras, Paraíba.

2 Mestra em Sistemas Agroindustriais pela UFCG. Pós-graduanda em Cardiologia. Graduada em Medicina pela Faculdade de Medicina Nova Esperança FAMENE (2011). Especialização em Preceptoria de Residência Médica no SUS pelo Hospital Sírio Libanês. Especialização em Medicina Intensiva pela Faculdade Redentor, Rio de Janeiro. Atualmente Médica Intervencionista do Serviço de Atendimento Móvel de Urgência (SAMU), Médica diarista do Hospital Padre Alfredo Barbosa), atua na Faculdade Santa Maria como Docente do curso de medicina, coordenadora do Internato Médico e responsável técnica da Clínica Santa Maria, Cajazeiras, Paraíba.

3 Médica com graduação em Medicina pela Universidade Federal de Campina Grande (2008). Trabalhou por 8 meses como médica contratada do Programa de Saúde da Família da Prefeitura Municipal de Itapipoca de maio a dezembro de 2008. Concluiu Residência Médica pelo Programa de Pós-Graduação em Residência de Medicina de Família e Comunidade do Sistema Municipal de Saúde Escola da Prefeitura Municipal de Fortaleza com duração de 2 anos, tendo iniciado em janeiro de 2009 e concluído em janeiro de 2011. Possui Título de Especialista em Medicina de Família e Comunidade pela Associação Médica Brasileira. Exerceu a função de Médica de Família e Comunidade concursada do Programa de Saúde da Família da Prefeitura Municipal de Aurora-CE desde janeiro de 2011 a março de 2019, atualmente é médica concursada da Prefeitura Municipal de Barbalha-CE e, também, é supervisora do Programa Mais Médicos para o Brasil desde janeiro de 2014. Desde fevereiro de 2016 exerce a função de professora contratada da Faculdade Santa Maria (FSM) em Cajazeiras - PB das disciplinas Programa de Aprendizagem da Atenção Básica (PAAB) IV e VIII, Semiologia e Saúde do Idoso, e está na coordenação do Programa de Residência de Medicina de Família e Comunidade em Cajazeiras-PB.

${ }^{4}$ Pós-doutorado pela Universidade Federal de Campina Grande. Doutora em Ciências da Saúde pela Faculdade de Medicina do ABC. Docente da Faculdade Santa Maria, Cajazeiras, Paraíba.
} 
equipe multidisciplinar é responsável por englobar todos os aspectos de vida do usuário, como a sua espiritualidade, meio social, relações familiares, entre outros, que, juntos, servem de suporte para oferecer uma vida digna e se preparar para a hora da morte. Objetivo: Caracterizar a prática de cuidados paliativos na Atenção Primária à Saúde realizada por Médicos de Família e Comunidade. Método: Tratase de uma revisão bibliográfica, realizada no período de outubro de 2021 a janeiro de 2022 a partir das bases de dados Scientific Electronic Library Online (SciELO), Biblioteca Nacional em Saúde (BVS) e Literatura e National Library of Medicine (PubMed). Os critérios de inclusão dessa pesquisa foram artigos em inglês e português disponíveis nas bases de dados, publicados entre os anos de 2016 e 2021, com assunto cuidados paliativos na Atenção Primária. Resultados: Estudos afirmam que a oferta de cuidados paliativos está aquém do recomendado. Existe, atualmente, cerca de um serviço para cada 1.200.000 pessoas, ou seja, nem todos possuem o acesso como deveria ser. Além disso, muitos profissionais médicos não se sentem capacitados, e não possuem apoio multidisciplinar, como é recomendado. Assim como o Brasil, os demais países estão na luta para capacitação dos profissionais para implementação dos cuidados paliativos na Atenção Básica, definindo habilidades e critérios a serem seguidos para auxiliar na assistência. Conclusão: Com isso, pode-se concluir que os cuidados paliativos são uma prática pouco utilizada na Atenção Básica, e isto se dá, principalmente, pela falta de preparo da equipe em identificar e incluir no plano de cuidados do paciente, assim como de recursos.

Palavras-chave: Cuidados Paliativos. Atenção Primária à Saúde. Medicina de Família e Comunidade. Assistência Terminal.

ABSTRACT: Introduction: The current epidemiological scenario has resulted in the change of health care in a way that contemplates the entire health-disease process of individuals. In this sense, palliative care emerges as a strategy to continue providing care to people affected by diseases without cure, in order to ensure comfort and quality of life. The family is also included in this care, because it is the one who experiences more closely the changes in the individual's life. The multidisciplinary team is responsible for encompassing all aspects of the user's life, such as their spirituality, social environment, family relationships, among others, which together serve as support to offer a dignified life and prepare for the time of death. Objective: To characterize the practice of palliative care in Primary Health Care performed by Family and Community Physicians. Method: This is a bibliographic review, carried out from October 2021 to January 2022 from the Scientific Electronic Library Online (SciELO), National Health Library (VHL) and Literature and National Library of Medicine (PubMed) databases. The inclusion criteria of this research were articles in English and Portuguese available in databases published between 2016 and 2021 with palliative care in Primary Care. Results: Studies claim that the provision of palliative care is below the recommended. There is currently about one service for every 1,200,000 people, meaning not everyone has access as it should be. In addition, many medical professionals do not feel qualified and do not have multidisciplinary support as recommended. Like Brazil, the other countries are in the fight to train professionals to implement palliative care in Primary Care, defining skills 
and criteria to be followed to assist in care. Conclusion: Therefore, it can be concluded that palliative care is a practice little used in Primary Care and this is mainly due to the team's lack of preparation to identify and include in the patient's care plan, as well as resources.

Keywords: Palliative care. Primary Health Care. Family and Community Medicine. Terminal Care. 


\section{INTRODUÇÃO}

Com a redução da natalidade e da mortalidade por doenças infecciosas, somado ao avanço da tecnologia e da ciência, houve um aumento da expectativa de vida ao nascer. Em consequência disto, tem-se visto o envelhecimento da população brasileira e do aparecimento de Doenças Crônicas Não Transmissíveis (DCNT). A formação médica por anos foi tecnicista e despreparada para cuidar de pacientes com doenças incuráveis, porém, na metade do século XX, surgiram movimentos com foco na humanização da assistência, para que, assim, o sujeito seja visto na sua integralidade (RIBEIRO; POLES, 2019).

Neste sentido, no ano de 2017, a Política Nacional de Atenção Básica (PNAB) instituiu os Cuidados Paliativos (CP) como um dos seus campos de atuação. O CP tem como característica o cuidado humanizado em indivíduos com doenças que não possuem cura. Pode ser realizada no próprio domicílio do paciente, facilitando o vínculo e identificação do território, cultura e família envolvidos no processo saúdedoença (BRASIL, 2017; COSTA; OTHERO, 2014).

Os CPs correspondem à assistência de uma equipe multidisciplinar perante uma doença, aguda ou crônica, que ameace a vida, com finalidade de promover uma melhora na qualidade de vida do paciente e família a partir da detecção precoce, prevenção e alívio de dores e demais sintomas físicos, sociais, psicológicos e espirituais (BRASIL, 2018).

Considerando que este tipo de cuidado pode ser ofertado em qualquer ponto de atenção à saúde, como, por exemplo, na Atenção Domiciliar, Atenção Ambulatorial, Urgência e Emergência, Atenção Hospitalar, em alguns países já se discute que na Atenção Primária (AP) é o melhor ambiente para oferta dos CPs, justamente pela maior proximidade da comunidade e por possibilitar maior conhecimento das características emocionais, culturais, sociais e o suporte que o paciente possui (BRASIL, 2018; RIBEIRO; POLES, 2019). 
A Atenção Primária é tida como a porta de entrada do usuário ao sistema de saúde, e tem como objetivo ofertar um cuidado integral e referenciar para os demais níveis de atenção, como a Atenção Secundária e Terciária. Foi criada em 1994 a partir do Programa de Agentes Comunitários (PACS), para reorganizar o modelo assistencial da época, seguindo os princípios e diretrizes do Sistema Único de Saúde (SOUZA et al., 2017; SILVA, 2014).

Considerando que esse tipo de cuidado pode ser ofertado em diferentes ambientes, e a Atenção Primária à Saúde tida como uma possibilidade, ainda existem dificuldades na implementação desse tipo de assistência, como a falta de recursos e insumos, capacitação profissional, demora na marcação de exames, ausência de medicações, entre outros (RIBEIRO; POLES, 2019).

Pois, apesar do avanço científico, ainda existe uma deficiência na educação médica de formar profissionais tecnicistas e mecânicos, sem preparo para cuidar de um paciente com doença grave e sem cura (RIBEIRO; POLES, 2019).

Contudo, o Médico de Família e Comunidade (MFC) surge como um dos profissionais essenciais para atender dentro desse contexto, responsável por articular o cuidado entre as demais redes de atenção à saúde e garantir o acesso aos serviços. A oferta de CPs impacta diretamente na redução de ocorrências em unidades de emergência, menor tempo de hospitalização e, consequentemente, melhor qualidade de vida (WONG et al., 2016; GUSSO; LOPES, 2018).

A Sociedade Brasileira de Medicina de Família e Comunidade (SBMFC) desenvolveu um currículo, que contempla ações esperadas do médico atuante nesta área, baseado no quadro clínico do paciente. Dentre essas competências está o manejo da dor em pacientes terminais, orientações que podem ser fornecidas aos familiares relacionadas à morte do paciente, manejo para intercorrências de cuidados paliativos e como abordar o luto (SBMFC, 2015).

Neste sentido, surgiu o interesse em trabalhar essa temática a partir das vivências na Atenção Primária à Saúde, em que pudemos acompanhar diversos pacientes em condições que necessitavam cuidados paliativos, e de conhecer, de maneira mais aprofundada, como esta prática é implementada na rede de saúde.

Este estudo é relevante, por possibilitar um conhecimento acerca da atuação médica dentro do contexto da Atenção Básica de Saúde em cuidados paliativos, e 
como esses profissionais visualizam esse tipo de assistência e a realizam, levando em consideração a patologia de cada paciente e suas condições socioeconômicas. Dessa forma, o objetivo do presente estudo é caracterizar a prática de cuidados paliativos na Atenção Primária à Saúde realizada por Médicos de Família e Comunidade.

\section{MÉTODO}

Estudo com abordagem qualitativa com a utilização do método de revisão da literatura realizada a partir das bases de dados Scientific Electronic Library Online (SciELO), Biblioteca Nacional em Saúde (BVS) e Literatura e National Library of Medicine (PubMed), no período entre outubro de 2021 a janeiro de 2022.

A revisão da literatura é um método fundamental para a elaboração de trabalhos acadêmicos e científicos, evita a duplicação de pesquisas e, em muitos casos, reaproveita estudos e aplica em outros contextos. Além disso, contribui para a identificação de possíveis falhas em estudos já realizados, e propõe temas e hipóteses relevantes para o campo científico (BAEK et al., 2018).

Os Descritores em Ciências da Saúde (DeCS) utilizados foram: Cuidados Paliativos, Atenção Primária à Saúde, Medicina de Família e Comunidade e Assistência Terminal.

Para busca inicial foi feito o cruzamento dos DeCs, aplicado os critérios de seleção e realizada uma leitura flutuante dos títulos e resumos dos artigos encontrados, descartando as publicações que não estavam relacionadas ao objetivo do estudo. Após essa etapa, foram selecionados alguns artigos para uma leitura mais detalhada de análise do conteúdo e contribuição com a pesquisa.

A pesquisa foi realizada nas bases de dados citadas a partir do cruzamento dos descritores e dos critérios de inclusão e exclusão definidos. Foram encontrados 21 artigos na Scielo, 14 artigos na BVS e 73 na PubMed. Após leitura do título e resumo, para identificar o tema principal, foram selecionados 29 artigos para uma leitura mais detalhada, para verificar se contemplava os objetivos da pesquisa. 
Apesar da alta quantidade de artigos encontrados, a grande maioria abordava a aplicação dos cuidados paliativos em outros ambientes que não a Atenção Primária à Saúde, por isso que a seleção foi baixa.

Os critérios de inclusão da revisão foram: artigos em inglês e português disponíveis nas bases de dados publicados, entre os anos de 2016 e 2021, com assunto cuidados paliativos na Atenção Primária. Foram excluídos estudos que tivessem artigos com resumos não disponíveis, teses e dissertação, e que abordem a percepção dos cuidados paliativos por cuidadores, e de pacientes em cuidado paliativo na atenção especializada.

\section{RESULTADOS E DISCUSSÃO}

Foram selecionados vinte e nove artigos pelo título e resumo para uma leitura mais ampla acerca dos cuidados paliativos em Atenção Primária à Saúde e, destes, doze contemplaram os objetivos e critérios de inclusão e exclusão definidos pela pesquisa. Sendo quatro artigos encontrados na BVS, cinco na SciELO e três na PubMed.

O século XIX foi marcado pelo crescimento na área das ciências e tecnologia, e pelo momento em que igreja diminuía o seu poder sobre a sociedade. Neste sentido, surgiram os primeiros hospitais que ofertavam cuidado para salvar a vida dos pacientes e, ao mesmo tempo, ensinavam a ciência médica conhecida através das guerras. Nesse meio tempo, surgiam instituições que preparavam pessoas e ofertavam conforto espiritual para a morte próxima (FLORIANI; SCHRAMM, 2007).

O cuidado aos pacientes terminais, acometidos por doenças que não possuem cura, são questionados a partir do momento em que acontece uma mudança no processo de morrer e adoecer, principalmente diante de uma transição demográfica e epidemiológica, em que há o aumento das doenças crônicas degenerativas e redução das doenças infecciosas e parasitárias (JUSTINO et al., 2020). 
Neste sentido, em 1990, o conceito de Cuidados Paliativos (CP) foi publicado pela Organização Mundial da Saúde (OMS) como sendo um conjunto de cuidados holísticos, ofertados por uma equipe multidisciplinar às pessoas que estão no final da vida, com objetivo de melhorar a sua qualidade de vida e a dos seus familiares. Em agosto de 2017, o conceito foi reformulado, e a OMS ressaltou a importância de implementar os CPs para todas as idades, e enfatizou a inclusão da família neste processo (WHO, 2017).

Segundo Costa e colaboradores (2020), o crescente número de pessoas acometidas pelas doenças crônicas, incapacitantes, incuráveis e que trazem ameaça à vida nos dias atuais, é motivo suficiente para o aumento das investigações no contexto da oferta de CPs dentro da Atenção Primária à Saúde (APS).

O Sistema Único de Saúde (SUS), dentro da sua vasta oferta de cuidados aos usuários através dos programas e políticas públicas, será essencial durante essa transição epidemiológica, em que há também um aumento no número de idosos e de demanda mais complexa dos serviços de saúde (DANTAS et al., 2017).

Um grande desafio para a prática apropriada do CP é a avaliação do paciente e identificação dos parâmetros que embasem cientificamente a assistência. É preciso aliar os conhecimentos técnicos e humanos, com envolvimento da família e paciente, nas tomadas de decisão. O profissional precisa realizar uma anamnese completa e conhecer a cronologia da doença, assim como realizar uma avaliação funcional do paciente (BRASIL, 2020).

Um estudo realizado por Mattos e Derech (2020), em 34 cidades brasileiras com Médicos de Família e Comunidade (MFC) da APS, buscou investigar a formação destes para aplicação dos CPs, e a maioria dos entrevistados relatou não ter especialização e não cursar nenhuma disciplina durante a graduação sobre CP. Este achado demonstra que ainda possuem profissionais que não recebem instrução adequada para utilização desse cuidado.

Os MFC são habilitados para ofertar um cuidado centrado na pessoa, independente do momento de vida, quadro clínico ou sofrimento. No entanto, alguns ainda se sentem incapacitados na oferta dos $\mathrm{CPs}$, pois não possuem capacitação adequada e oportunidade de aprendizado durante a graduação (SOCIEDADE 
BRASILEIRA DE MEDICINA DE FAMÍLIA E COMUNIDADE, 2015; GRYSCHEK; PEREIRA; HIDALGO, 2020).

Neste sentido, considerando o potencial do médico de família dentro da APS, a Sociedade Brasileira de Medicina de Família e Comunidade, em 2015, elaborou o Currículo Baseado em Competências (CBC), e incluiu os CPs para auxiliar na formação de médicos residentes. O documento é dividido em quatro níveis: prérequisito, essencial, desejável e avançado (SBM, 2015).

O primeiro fala sobre as competências que o profissional deveria desenvolver durante a sua especialização, durante a graduação médica. $O$ segundo ao aprendizado mínimo exigido ao término da residência. Os dois últimos níveis se referem às competências que todo profissional deveria adquirir, com excelência, em determinada área. Esse reconhecimento da SBM como competência é importante para a capacitação dos profissionais e oferta do CP no Brasil (GRYSCHEK; PEREIRA; HIDALGO, 2020).

Isso porque, apesar da extensa gama de serviços de saúde no Brasil, a Academia Nacional de Cuidados Paliativos identificou apenas 177 serviços que ofertam cuidados paliativos. Destes, 130 são ofertados em hospitais, e, aproximadamente, 9 funcionam o modelo hospice. O modelo hospice diz respeito ao cuidado a pacientes com doenças letais, principalmente quando se tornam incuráveis, e a terapia paliativa é a opção (ACADEMIA NACIONAL DE CUIDADOS PALIATIVOS, 2018).

O estudo ainda identificou que a maioria desses está localizada na Região Sudeste. Considerando a população brasileira, seria um serviço para cada 1.200.00 pessoas, ou seja, nem todos os indivíduos que necessitam do CP possuem acesso (ACADEMIA NACIONAL DE CUIDADOS PALIATIVOS, 2018).

Diante deste contexto, diversos autores enfatizam que a educação e treinamento de novos profissionais é a alternativa para a expansão da oferta deste modelo de cuidado no país. Principalmente para o MFC, conforme orientações do CBC, para atuação dentro da APS, pois é a principal porta de entrada da população ao sistema de saúde. A partir do momento em que a oferta do CP for aumentada, exigirá que os profissionais estejam ainda mais capacitados para aplicá-los com excelência (PAVLIČ et al., 2019; GRYSCHEK; PEREIRA; HIDALGO, 2020). 
Ainda segundo Gryschek, Pereira e Hidalgo (2020), é preciso que haja uma melhoria e uma revisão periódica do CBC, para contemplar avanços e necessidades vistas nas demandas atendidas, além de incluir a disseminação de informação acerca dos Cuidados Paliativos para a sociedade.

O SUS, quando foi estruturado, não incorporou em seu escopo a oferta dos CPs, porém, em 2017, a Política Nacional de Atenção Básica o instituiu como um dos seus campos de atuação. No entanto, ainda é possível observar falhas na gestão e estrutura do sistema, principalmente no que concerne à capacitação dos profissionais e de ações voltadas aos pacientes e família, de forma específica (SILVA; NIETSCHE; COGO, 2021).

Além disso, de acordo com os autores Mattos e Derech (2020), existe dificuldade do MFC atuar de maneira multidisciplinar, tendo em vista a falta de capacitação de diversos profissionais sobre $\mathrm{CP}$, principalmente com relação ao suporte psicológico e emocional aos pacientes e familiares, que é recomendado nas diretrizes na Resolução nำ 41, de 31 de outubro de 2018.

A Resolução no 41, de 31 de outubro de 2018, dispõe sobre a organização dos cuidados paliativos à luz dos cuidados integrados no contexto do SUS, realizado através da Rede de Atenção à Saúde (RAS). A resolução aborda os objetivos, princípios norteadores, financiamento e ambientes em que o CP pode ser ofertado dentro da rede (BRASIL, 2018).

A APS é, sem dúvida, o ambiente ideal para contribuir com os pacientes que estão no fim da vida, de forma a ofertar um cuidado sistêmico, individual e humanizado com foco na autonomia. Por isso que é necessário, para assistir um paciente em cuidados paliativos, um olhar multidimensional da equipe (VIEIRA et al., 2016).

O estudo de Vieira e colaboradores (2016) enfatiza a importância de englobar o cuidado integral. O estudo baseou-se no caso clínico de uma paciente que esgotou as possibilidades de tratamento, e a espiritualidade foi utilizada como recurso de aceitação da doença tanto para a paciente, quanto para a família. A partir disso, houve melhora na qualidade de vida com aceitação da dieta, participação de grupos da igreja e fortalecimento de laços familiares até o fim da sua vida. A 
espiritualidade pode ser buscada através da religião, da música ou de questões pessoais ou científicas, mas que traz sentimento de paz e conforto.

Com relação à percepção dos médicos sobre CP, Ribeiro e Poles (2019) realizaram um estudo com 16 médicos, atuantes na Unidade Saúde da Família, em um município do Sul de Minas. A maioria dos médicos possui conhecimento básico acerca dos cuidados paliativos, mas restringe apenas ao alívio dos sintomas físicos. A minoria aborda a inclusão da família, a espiritualidade, fatores emocionais, dentre outros, que podem ser extremamente importantes no enfrentamento de uma doença sem cura.

Este estudo ainda corrobora com os achados acima de que poucos médicos possuem contato com esta área e com disciplinas que abordem o processo de morte e o morrer durante a formação médica. Por isso que, ao serem questionados se se sentem preparados para atender um paciente em cuidados paliativos, a maioria respondeu que não ou não totalmente. Dentre os diversos motivos, o principal é a falha na formação acadêmica (MATTOS; DERECH, 2020).

Outro ponto bastante importante abordado no estudo de Ribeiro e Poles (2019) é a baixa demanda desse cuidado, apontada pelos entrevistados médicos na realidade da Atenção Primária à Saúde. Todavia, isso pode ser explicado pela dificuldade dos profissionais de identificar a necessidade de um paciente voltada ao cuidado paliativo, ocasionada pela pouca experiência e conhecimento.

É importante que o serviço de saúde e os profissionais conheçam quais são os critérios de elegibilidade para oferta do cuidado paliativo, portanto, neste perfil, são inclusas as doenças progressivas, incuráveis e avançadas, doenças com poucas possibilidades de resposta terapêutica diante de um tratamento curativo, prognóstico de vida estimado em menos de seis meses, casos de evolução clínica oscilante, caracterizado por crises ou reagudizações, dentre outras (INSTITUTO DE SAÚDE E GESTÃO HOSPITALAR, 2014).

Salienta-se que, apesar dos medos e dificuldades dos profissionais em discutir o prognóstico ou terminalidade de uma doença com o paciente ou família, não é menos ou mais difícil do que discutir sobre o diagnóstico de doenças raras ou mudança de tratamento. É considerado um dever do profissional, altamente discutido em âmbito jurídico, de proporcionar a melhor qualidade de vida possível ao 
paciente, diante dos recursos e técnicas existentes (INSTITUTO DE SAÚDE E GESTÃO HOSPITALAR, 2014).

Dentre os usuários menos propensos a receber cuidados paliativos estão os homens. Isso ocorre pela dificuldade de procura do homem pelo serviço de saúde e pela opção de continuar combatendo a doença ao invés de escolher o tratamento paliativo, pois isso é visto como sinônimo de "luta" para a sociedade (SAEED et al., 2018).

A realidade do Brasil não é diferente dos demais países. Na Índia, por exemplo, foi instituído um programa de educação continuada na Unidade Básica de Saúde, para capacitar os profissionais médicos na oferta dos cuidados paliativos. No país, a demanda por esse tipo de cuidado tem tido uma melhor aceitação, principalmente entre os profissionais, devido ao impacto que esse possui na qualidade de vida do indivíduo, capaz de reduzir, por exemplo, a quantidade de intervenções invasivas inadequadas no fim da vida (ATREYA et al., 2019).

Inclusive, Atreya e demais autores (2019), em seu artigo, abordam que, nos últimos anos, na Índia, foram realizadas conferências com Médicos de Família e Comunidade e de Cuidados Paliativos, para determinar competências essenciais para o profissional prestar esse tipo de assistência na comunidade, elencando as habilidades, conhecimentos básicos, atitudes necessárias para prestar os cuidados paliativos, assim como identificar as pessoas que necessitam desse tipo de cuidado, apoio às necessidades psicossociais, entre outros aspectos.

Diversas terapias são encontradas como forma de buscar resultados positivos na vida dos indivíduos que estão em tratamento com cuidados paliativos. Santos e colaborares (2020) buscaram, em sua pesquisa, avaliar os efeitos da atividade clown na qualidade de vida, ansiedade, depressão, suporte e apoio social em pacientes elegíveis ao CP atendidos na Atenção Primária.

A terapia clown, também chamada de terapia do riso, é utilizada como recurso terapêutico para melhora da condição emocional, que se adapta às condições impostas pela doença, como a dor, angústia, tristeza, já que o palhaço tem uma forma de ver as coisas com outra perspectiva. Foi identificada a primeira vez na época de Hipócrates, pois os médicos acreditavam que o humor reproduzia efeitos positivos no processo saúde-doença (SANTOS et al., 2020; PIRES et al., 2015). 
Foram realizadas visitas pelos palhaços, no domicílio do paciente em cuidados paliativos, e isso demonstrou uma melhora nas relações interpessoais, fortalecendo a relação paciente-família-profissionais. Apesar disto, houve uma piora nos sintomas depressivos e ansiosos, que pode estar associada à ansiedade de esperar as visitas, pela própria condição de saúde, bem como pelo término das ações (SANTOS, et al., 2020). Sendo assim, é imprescindível que os profissionais busquem ofertar o melhor para o paciente e sua família, mesmo diante de um quadro clínico, de forma a considerar a oferecer condições necessárias para uma morte digna.

\section{CONCLUSÃO}

Diante do exposto, foi possível identificar a importância do médico de família e comunidade, dentro de uma equipe multidisciplinar, na oferta dos cuidados paliativos dentro do contexto da Atenção Primária à Saúde. O cuidado paliativo tem sido cada vez mais implementado na vasta gama de serviços ofertados pelo SUS, porém foi identificado que nem todos os profissionais possuem segurança para implementar um plano de cuidado paliativo.

Ficou evidente, diante da pesquisa, que o medo e receio dos profissionais médicos são evidenciados pela baixa demanda, aliados ao próprio profissional de buscar conhecer e oferecer o melhor para o paciente. Sabe-se que o cuidado paliativo pode elevar a qualidade de vida do paciente consideravelmente, e que os impactos sociais, econômicos e psicológicos que uma doença incurável acarreta na vida do paciente e família são enormes.

Então, alguns cuidados que podem ser ofertados, além do controle da dor, é utilizar da espiritualidade, da música, das relações sociais, como forma de oferecer conforto e alívio dos sintomas. Somado ao cuidado ao paciente, a família também precisa ser levada em consideração, para que se torne uma rede de apoio fortificada e consciente do quadro clínico do paciente, ajudando-o a superar as dificuldades encontradas no meio do tratamento paliativo e aceitação da doença. 
A terapia clown foi citada por alguns autores, porém ainda são poucos os estudos que se aprofundam na temática, principalmente voltada à sua aplicação da APS. No entanto, demonstrou benefícios na qualidade de vida do paciente e achados importantes, como prejuízo nos sintomas ansiosos e depressivos, que podem ser mais estudados, a fim de melhorar sua aplicação.

Além disso, é preciso que haja novos estudos com relação ao papel do profissional médico dentro da Atenção Básica, de forma a disseminar essa prática entre os próprios profissionais e a sociedade, a fim de possibilitar uma morte digna ao paciente e conforto familiar.

Como limitação do estudo está a dificuldade em encontrar estudos que abordem a atuação médica em cuidados paliativos dentro da Atenção Básica de Saúde, pois a maioria dos estudos aborda o contexto do cuidado em consultórios especializados ou hospitais. 


\section{REFERÊNCIAS BIBLIOGRÁFICAS}

ACADEMIA NACIONAL DE CUIDADOS PALIATIVOS. Análise Situacional e Recomendações para Estruturação de Programas de Cuidados Paliativos no Brasil. São Paulo; 2018.

ATREYA, S., et al. Primary palliative care competency framework for primary care and family physicians in India-Collaborative work by Indian Association of Palliative Care and Academy of Family Physicians of India. Journal of family medicine and primary care, v. 8 , n. 8 , p. 2563 , 2019.

BAEK, S. et al. The most downloaded and most cited articles in radiology journals: a comparative bibliometric analysis. European Radiology, v. 28, n. 11, p. 4832-4838, 2018.

BRASIL. Ministério da Saúde. Política Nacional de Atenção Básica. Departamento de Atenção Básica. (2017). Portaria no. 2.436 de 21 de setembro de 2017. Brasília, 2017.

BRASIL. Ministério da Saúde (BR), Resolução № 41, de 31 de outubro de 2018. Dispõe sobre as diretrizes para a organização dos cuidados paliativos, à luz dos cuidados continuados integrados, no âmbito Sistema Único de Saúde (SUS). Brasília (DF): Diário Oficial da União; 2018.

BRASIL. Ministério da Saúde. Secretaria de Atenção Especializada à Saúde. Departamento de Atenção Hospitalar, Domiciliar e de Urgência. Atenção Domiciliar na Atenção Primária à Saúde - Brasília: Ministério da Saúde, 2020.

COSTA, A. P. P.; OTHERO, M. B. Conceitos, princípios e formação em Cuidados Paliativos. Costa APP, Otheroff MB. Reabilitação em cuidados paliativos. Loures: Lusodidacta, p. 2336, 2014.

COSTA, L. F., et al. Resposta ao artigo: Cuidados paliativos providos por médicos de família e comunidade na atenção primária à saúde brasileira. Revista Brasileira de Medicina de Família e Comunidade, v. 15, n. 42, p. 2469-2469, 2020.

DANTAS, I. C., et al. Perfil de morbimortalidade e os desafios para a atenção domiciliar do idoso brasileiro. Revista Kairós: Gerontologia, v. 20, n. 1, p. 93-108, 2017.

FLORIANI, C. A.; SCHRAMM, F. R. Desafios morais e operacionais da inclusão dos cuidados paliativos na rede de atenção básica. Cadernos de Saúde Pública, v. 23, n. 9, p. 2072-2080, 2007.

GRYSCHEK, G.; PEREIRA, E. A. L.; HIDALGO, G. Médicos de Família e Cuidados Paliativos: contribuições ao currículo baseado em competências. Revista Brasileira de Medicina de Família e Comunidade, v. 15, n. 42, p. 2012-2012, 2020.

GUSSO, G.; LOPES, J. M. C. Tratado de Medicina de Família e Comunidade: princípios, formação e prática. $2^{\mathrm{a}}$ ed. Porto Alegre: Artmed; 2018. $2432 \mathrm{p}$.

INSTITUTO DE SAÚDE E GESTÃO HOSPITALAR. PROTOCOLOS ISGH. CUIDADOS PALIATIVOS, 2014.

JUSTINO, E. T. et al. Cuidados paliativos en la atención primaria a la salud: scoping review. Revista Latino-Americana de Enfermagem, v. 28, 2020.

MATTOS, C. W.; DERECH, R. D. Cuidados paliativos providos por médicos de família e 
comunidade na atenção primária à saúde brasileira. Revista Brasileira de Medicina de Família e Comunidade, v. 15, n. 42, p. 2094-2094, 2020.

PAVLIČ, D. R., et al. Palliative care in primary care: European Forum for Primary Care position paper. Primary health care research \& development, v. 20, 2019.

PIRES, W. G. B., et al. Efeito da intervenção clown no padrão de depressão de idosos em instituição de longa permanência. Ciencia y Enfermeria, v. 21, n. 2, p. 99-111, 2015.

RIBEIRO, J.R.; POLES, K. Cuidados paliativos: prática dos médicos da estratégia saúde da família. Revista Brasileira de Educação Médica, v. 43, p. 62-72, 2019.

SANTOS, F. R., et al. Efeitos de atividades clown em pacientes elegíveis para cuidados paliativos na atenção primária à saúde. Revista Brasileira de Enfermagem, v. 74, 2021.

SAEED, F., et al. Preference for palliative care in cancer patients: are men and women alike? Journal of pain and symptom management, v. 56, n. 1, p. 1-6. e1, 2018.

SILVA, M. L. S. R. O papel do profissional da Atenção Primária à Saúde em cuidados paliativos. Revista Brasileira de Medicina de Família e Comunidade, v. 9, n. 30, p. 45-53, 2014.

SILVA, T. C.; NIETSCHE, E. A.; COGO, S. B. Cuidados paliativos na Atenção Primária à Saúde: revisão integrativa de literatura. Revista Brasileira de Enfermagem, v. 75, 2021.

SOCIEDADE BRASILEIRA DE MEDICINA DE FAMÍLIA E COMUNIDADE. Currículo baseado em competências para Medicina de Família e Comunidade. 2015.

VIEIRA, R. R., et al. Vida e morte na atenção primária à saúde: reflexões sobre a vivência do médico de família e comunidade ante a finitude da vida. Revista Brasileira de Medicina de Família e Comunidade, v. 11, n. 38, p. 1-7, 2016.

WONG, F. K. Y., et al. Efeitos de um modelo transicional de cuidados paliativos em pacientes com insuficiência cardíaca em estágio terminal: um ensaio clínico randomizado. Coração, v. 102, n. 14, pág. 1100-1108, 2016.

World Health Organization (WHO). Palliative Care Fact Sheet. World Health Organization, Genebra; 2017. 